\title{
A Prospective Study to Evaluate the Effect of Paclitaxel on Cardiac Ejection Fraction
}

\author{
Mohammed Osman ${ }^{a}$ Mohammad Elkady ${ }^{b}$ \\ ${ }^{a}$ General Organization for Teaching Hospital, Cairo, Egypt; \\ ${ }^{\mathrm{b}}$ Ain Shams University, Cairo, Egypt
}

\section{Keywords \\ Paclitaxel · Cardiotoxicity · EF · Echocardiography · CHF}

\section{Summary}

Background: The primary objective of the study was to evaluate the long-term changes in ejection fraction (EF) associated with paclitaxel infusion. Methods: 50 patients were enrolled in this prospective study between 2011 and 2015. The study design included frequent follow-up visits to the clinic, EF evaluation at baseline, and regular EF assessment by echocardiography for 30 months after treatment. Results: The median baseline EF was $60 \%$ (95\% confidence interval (Cl) 50-80\%). At 30 months, the median EF was $48 \%$ (95\% Cl 40-60\%; $p=0.03$ ). During the 30-month follow-up, $10(20 \%)$ patients developed grade 1 and 2 cardiotoxicities; none developed grade 3 or 4 cardiotoxicities. Furthermore, paclitaxel cardiotoxicity increased among patients with high-risk features including associated diabetes mellitus, hypertension, prior radiotherapy to the chest wall, performance status of 2, and age $>60$ years. Conclusion: Paclitaxel has cardiotoxic effects. Careful monitoring of cardiac function during and after paclitaxel infusion is required in patients with high-risk features.

(C) 2017 S. Karger GmbH, Freiburg

\section{Introduction}

Paclitaxel was discovered in 1962 as a mitotic spindle inhibitor. Since then, paclitaxel has demonstrated activity in many cancers including breast, ovarian, gastric, endometrial, and lung cancers [1-4].
With recent advances in cancer management, the number of cancer survivors has doubled in the last 3 decades [5]. Cardiotoxicity is one of the most serious adverse effects of chemotherapy, resulting in a rise in morbidity and mortality. Cardiac dysfunction associated with chemotherapy can be an acute, subacute, or chronic side effect. Acute or subacute cardiotoxicity develops any time from the initiation of treatment up to 2-4 weeks after the completion of therapy and is characterized by reversible arrhythmias, abnormalities in ventricular repolarization, prolongation of QT interval, acute coronary syndrome, pericardial reaction, and alteration in myocardial function. Chronic cardiotoxicity occurs 4 weeks after treatment and is divided into early cardiotoxicity, which appears within the first year after treatment, and late cardiotoxicity, which appears more than 1 year after chemotherapy. Chronic cardiotoxicity includes reversible or irreversible cardiac (systolic or diastolic) dysfunction, which can lead to irreversible heart failure and even death [6-8].

Paclitaxel-induced cardiotoxicity occurs either indirectly following a massive histamine release with subsequent conduction disturbances and arrhythmia, or through direct myocardial damage via an effect on subcellular organelles, which may induce congestive heart failure (CHF) $[9,10]$. Studies showed that the most important acute arrhythmogenic effect of paclitaxel is QT interval prolongation, followed by bradycardia, then atrial fibrillation [11, 12]. CHF is defined as a reduction in ejection fraction (EF) to $\leq 50 \%$ with symptoms, or an asymptomatic reduction in $\mathrm{EF}$ to $\leq 45 \%[13,14]$.

The primary objective of this study was to evaluate the longterm changes in EF associated with paclitaxel infusion. The secondary objectives were to correlate this change with predetermined factors and to assess the initial symptoms of $\mathrm{CHF}$ associated with a decline in EF.

\section{KARGER}

๑ 2017 S. Karger GmbH, Freiburg
Dr. Mohammed Osman

General Organization for Teaching Hospital

Kasr Elaini Street

Cairo, Egypt

mmoneam@hotmail.com 


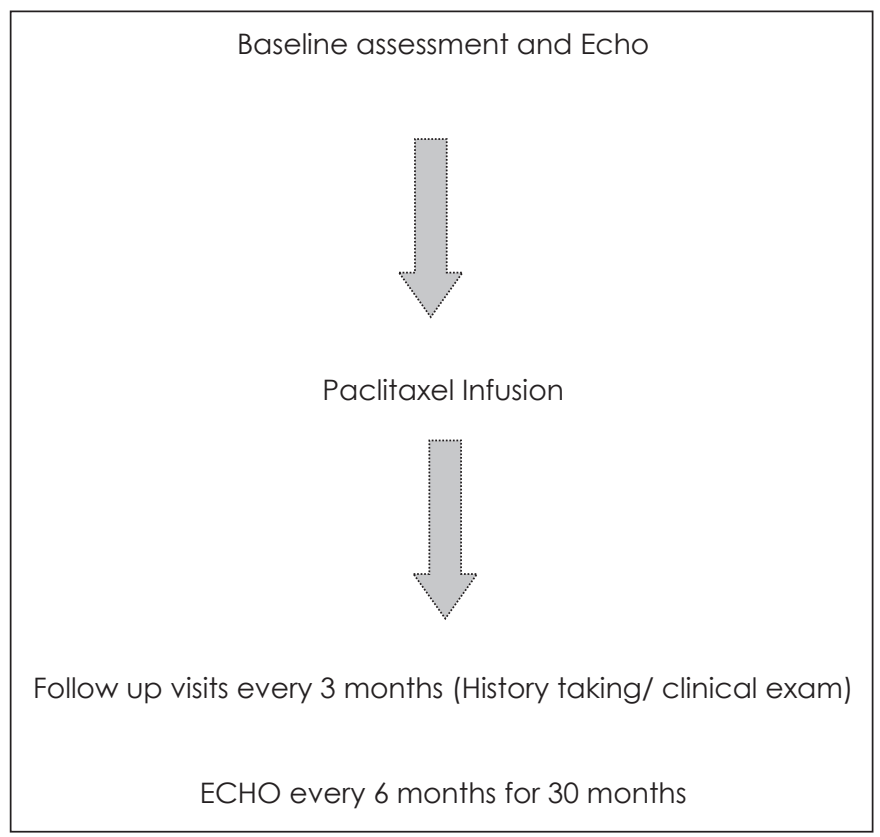

Fig. 1. Design of the current trial.

\section{Patients and Methods}

\section{Patients}

Patients were enrolled in the current prospective study if they fulfilled the inclusion criteria: Patients had to have histologically proven gynecological malignancies including breast malignancies in either the neoadjuvant, adjuvant, or metastatic/recurrent setting. Patients had to be chemo-naïve or have previously received only 1 line of chemotherapy which had to be non-cardiotoxic and include agents not known to decrease the EF. A period of $\geq 2$ years must have passed since the last cycle, and a period of 2 years must have passed since the end of the last radiotherapy to the chest wall. Patients should have received paclitaxel as part of their chemotherapy protocol, either as a single agent or in combination. When given in combination, paclitaxel had to be combined with other non-cardiotoxic agents. In addition, patients had to be 18-65 years of age, with an Eastern Cooperative Oncology Group (ECOG) performance status of 0-2. Patients were to not have any associated cardiac comorbidities, with the exception of hypertension, diabetes mellitus, and hypercholesterolemia. The patients' EF at baseline had to be $\geq 50$ by echocardiography. Finally, patients had to have adequate baseline hematological, renal (creatinine up to $1.5 \times$ upper normal limit), and hepatic (normal bilirubin) profiles.

Patients from 3 educational hospitals in Egypt were enrolled, including the Oncology Units of Ain Shams University Hospitals, El Sahel Teaching Hospital, and Ismailia Oncology Teaching Hospital.

\section{Study Design}

After ethical approval, patients were enrolled in the current prospective trial. The study design is shown in figure 1 .

\section{Follow-Up}

After finishing the treatment protocol, patients were followed up by regular clinic visits as per the National Comprehensive Cancer Network (NCCN) guidelines for follow-up of patients after cancer treatment. This included history taking and clinical examination every 3 months in the first 2 years, every 6 months for the following 3 years, and annually thereafter. At each visit, patients were evaluated for symptoms of CHF including shortness of breath (SOB), orthopnea (SOB upon lying flat), lower limb swelling, palpitation, and new fatigue. Clinical examinations were carried out to detect signs of cardiac dysfunction (basal lung crackles, gallop, and lower limb edema) [15].
Table 1. Changes in ejection fraction (EF) according to NCI-CTC version 2

\begin{tabular}{ll}
\hline Grade & Features \\
\hline 0 & up to $5 \%$ decrease in EF from baseline \\
1 & asymptomatic decline in $\mathrm{EF}$ of $>5 \%$ but $<20 \%$ from baseline \\
2 & asymptomatic decline in $\mathrm{EF} \geq 20 \%$ from baseline \\
3 & Congestive heart failure (CHF) responsive to treatment \\
4 & severe or refractory CHF or requiring intubation \\
\hline
\end{tabular}

Table 2. Patient characteristics

\begin{tabular}{|c|c|}
\hline Characteristics & \\
\hline \multicolumn{2}{|l|}{ Age, years } \\
\hline Mean (median) & $49.5(50)$ \\
\hline $95 \%$ confidence interval & $25-65$ \\
\hline \multicolumn{2}{|l|}{ Sex, n (\%) } \\
\hline Female & $50(100)$ \\
\hline Male & $0(0)$ \\
\hline \multicolumn{2}{|l|}{ Performance status (ECOG), n (\%) } \\
\hline 0 & $3(60)$ \\
\hline 1 & $12(24)$ \\
\hline 2 & $8(16)$ \\
\hline \multicolumn{2}{|l|}{ Cancer type, n (\%) } \\
\hline Ovarian & $35(70)$ \\
\hline Breast & $10(20)$ \\
\hline Endometrial & $5(10)$ \\
\hline \multicolumn{2}{|l|}{ Paclitaxel, n (\%) } \\
\hline \multicolumn{2}{|l|}{ Single agent (initial dose) } \\
\hline Weekly $\left(80 \mathrm{mg} / \mathrm{m}^{2}\right)$ & $14(28)$ \\
\hline Thrice weekly $\left(175 \mathrm{mg} / \mathrm{m}^{2}\right)$ & $29(58)$ \\
\hline \multicolumn{2}{|l|}{ Combination } \\
\hline With carboplatin & $7(14)$ \\
\hline With other agents & $0(0)$ \\
\hline \multicolumn{2}{|l|}{ Paclitaxel setting, n (\%) } \\
\hline Neoadjuvant/adjuvant & $40(80)$ \\
\hline Metastatic/recurrent & $10(20)$ \\
\hline \multicolumn{2}{|l|}{ Prior treatment, $\mathrm{n}(\%)$} \\
\hline Chemotherapy & $5(10)$ \\
\hline Radiotherapy to chest wall & $5(10)$ \\
\hline \multicolumn{2}{|l|}{ Associated comorbidities, n (\%) } \\
\hline Hypertension & $20(40)$ \\
\hline Hypercholesterolemia & $18(36)$ \\
\hline Diabetes mellitus & $5(10)$ \\
\hline Ischemic heart disease & $3(6)$ \\
\hline Total, n (\%) & $50(100)$ \\
\hline
\end{tabular}

Serial monitoring of cardiac EF using 2-dimensional echocardiography every 6 months for 30 months was carried out. Cardiac toxicity was graded according to the National Cancer Institute (NCI) Common Toxicity Criteria (CTC) version 2 [16] (table 1).

\section{Statistical Analysis}

All calculations were carried out using prism-6 software for Windows ( $M i-$ crosoft, Redmond, WA, USA). All analyses were carried out using the intention-to-treat approach. Mean, median, and 95\% confidence interval (CI) values were used for the description of data. For comparisons between the predetermined high-risk factors (including age, ECOG PS, paclitaxel used (single agent or combination), prior chemotherapy, radiotherapy, and associated comorbidities) and cardiac toxicities, multivariate analysis for relative risk was applied. $p$ value was significant at $\leq 0.05$. 


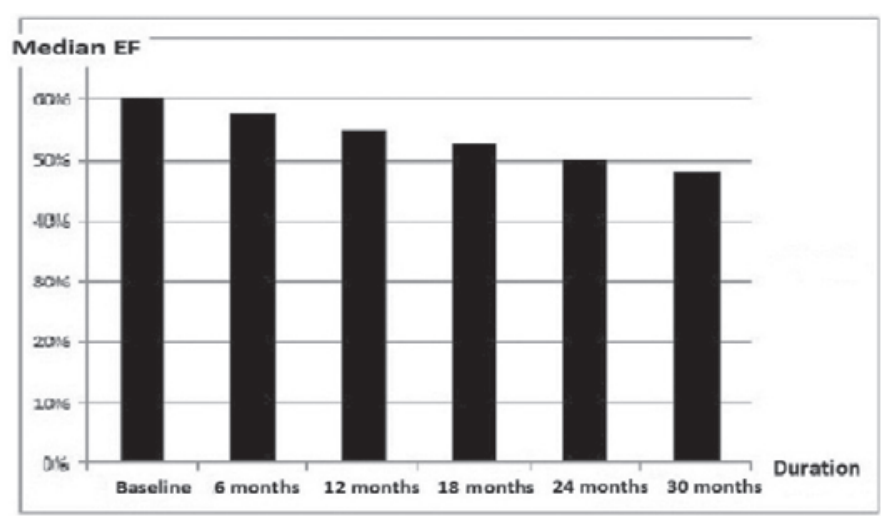

Fig. 2. Changes in median ejection fraction (EF) over time.

Table 3. Changes in ejection fraction (EF) over time during follow-up

\begin{tabular}{llll}
\hline $\begin{array}{l}\text { Time } \\
\text { (patients evaluated, \%) }\end{array}$ & $\begin{array}{l}\text { Median EF, } \\
\%\end{array}$ & $\begin{array}{l}95 \% \mathrm{CI}, \\
\%\end{array}$ & $\mathrm{p}$ \\
\hline Baseline (100) & 60 & $50-80$ & 0.03 \\
6 months (100) & 58 & $50-78$ & 0.03 \\
12 months (100) & 55 & $45-70$ & 0.03 \\
18 months (100) & 53 & $42-65$ & 0.03 \\
24 months (100) & 50 & $40-65$ & 0.03 \\
30 months (90) & 48 & $40-60$ & 0.04 \\
\hline
\end{tabular}

\section{Results}

Between May 2011 and June 2015, 50 patients were enrolled in the study. All fulfilled the study's inclusion criteria. Table 2 summarizes the patient characteristics.

All patients underwent baseline assessment and follow-up evaluation up to 24 months. 45 of the patients underwent evaluation at 6 months thereafter.

The median baseline EF was $60 \%$ (95\% CI 50-80\%). At 30 months, the median EF was $48 \%$ (95\% CI $40-60 \%)$. The changes in EF over time during follow-up are summarized in table 3 and figure $2(\mathrm{p}=0.03)$.

According to the NCI CTC version 2, during the 30-month follow-up, 8 patients developed grade 1 cardiac toxicity, and 2 developed grade 2 toxicity. No patient had grade 3 or 4 toxicity (table 4 ). Of the 10 patients who developed grade 1 and 2 cardiac toxicities, 4 developed these changes within 12 months, and the remaining 6 developed them between 12 and 24 months. None of the 50 patients evaluated developed new changes between 24 and 30 months. None of the 10 patients showed any new complaints related to cardiac dysfunction. All patients denied orthopnea, palpitations, lower limb swelling, or new fatigue. 2 (20\%) patients had new SOB with mild exertion at the time of diagnosis, which was intermittent in both cases. Furthermore, clinical examinations and
Table 4. Characteristics of patients who developed cardiac toxicities during the follow-up period

\begin{tabular}{|c|c|c|c|c|c|c|c|c|c|c|}
\hline & \multicolumn{8}{|c|}{ Grade 1} & \multicolumn{2}{|c|}{ Grade 2} \\
\hline & 1 & 2 & 3 & 4 & 5 & 6 & 7 & 8 & 1 & 2 \\
\hline \multicolumn{11}{|l|}{ Age, years } \\
\hline \multicolumn{11}{|l|}{$17-40$} \\
\hline $41-50$ & & & $\mathrm{x}$ & & $\mathrm{x}$ & & & & & \\
\hline $51-60$ & & & & $\mathrm{x}$ & & & & $\mathrm{x}$ & & \\
\hline $61-65$ & $\mathrm{x}$ & $\mathrm{x}$ & & & & $\mathrm{x}$ & $\mathrm{x}$ & & $\mathrm{x}$ & $\mathrm{x}$ \\
\hline \multicolumn{11}{|l|}{ Performance status } \\
\hline \multicolumn{11}{|l|}{0} \\
\hline 1 & $\mathrm{x}$ & & & $\mathrm{x}$ & & $\mathrm{x}$ & & $\mathrm{x}$ & & \\
\hline 2 & & $\mathrm{x}$ & $\mathrm{x}$ & & $\mathrm{x}$ & & $\mathrm{x}$ & & $\mathrm{x}$ & $\mathrm{x}$ \\
\hline \multicolumn{11}{|l|}{ Cancer type } \\
\hline Ovarian & $\mathrm{x}$ & & & & $\mathrm{x}$ & & $\mathrm{x}$ & $\mathrm{x}$ & $\mathrm{x}$ & \\
\hline Breast & & $\mathrm{x}$ & & $\mathrm{x}$ & & & & & & $\mathrm{x}$ \\
\hline Endometrial & & & $\mathrm{x}$ & & & $\mathrm{x}$ & & & & \\
\hline \multicolumn{11}{|l|}{ Paclitaxel } \\
\hline \multicolumn{11}{|l|}{ Single agent } \\
\hline Weekly & $\mathrm{x}$ & & & & $\mathrm{x}$ & & $\mathrm{x}$ & & & \\
\hline Every 3 weeks & & $\mathrm{x}$ & $\mathrm{x}$ & $\mathrm{x}$ & & & & & & $\mathrm{x}$ \\
\hline Combination & & & & & & $\mathrm{x}$ & & $\mathrm{x}$ & $\mathrm{x}$ & \\
\hline \multicolumn{11}{|l|}{ Paclitaxel setting } \\
\hline Neoadjuvant/adjuvant & & $\mathrm{x}$ & & & $\mathrm{x}$ & & $\mathrm{x}$ & $\mathrm{x}$ & & \\
\hline Metastatic/recurrent & $\mathrm{x}$ & & $\mathrm{x}$ & $\mathrm{x}$ & & $\mathrm{x}$ & & & $\mathrm{x}$ & $\mathrm{x}$ \\
\hline \multicolumn{11}{|l|}{ Prior treatment } \\
\hline Chemotherapy & & $\mathrm{x}$ & & & $\mathrm{x}$ & & & & $\mathrm{x}$ & \\
\hline Chest wall irradiation & & $\mathrm{x}$ & & $\mathrm{x}$ & & & & & & $\mathrm{x}$ \\
\hline \multicolumn{11}{|l|}{ Associated comorbidities } \\
\hline Hypertension & $\mathrm{x}$ & $\mathrm{x}$ & & $\mathrm{x}$ & & & & & $\mathrm{x}$ & $\mathrm{x}$ \\
\hline Hypercholesterolemia & $\mathrm{x}$ & & & & & & & & & $\mathrm{x}$ \\
\hline Diabetes mellitus & & $\mathrm{x}$ & & & $\mathrm{x}$ & & & & $\mathrm{x}$ & $\mathrm{x}$ \\
\hline Ischemic heart disease & & $\mathrm{x}$ & & & & & & & & \\
\hline
\end{tabular}


Table 5. Relative risk (RR) analysis to determine the correlation between cardiotoxicities and predetermined risk factors

\begin{tabular}{lll}
\hline Factor & $\mathrm{RR}$ & $\mathrm{p}$ \\
\hline Age > 60 vs. < 60 years & 0.7 & 0.04 \\
Performance status 2 vs. 0-1 & 0.8 & 0.04 \\
Paclitaxel combination vs. single agent & 1.1 & 0.09 \\
Neoadjuvant/adjuvant vs. metastatic/recurrent setting & 1.3 & 0.05 \\
Prior treatment & & \\
$\quad$ Chemotherapy & 0.9 & 0.1 \\
$\quad$ Radiotherapy & 0.85 & 0.055 \\
Associated comorbidities & & \\
$\quad$ Diabetes mellitus & 0.7 & 0.04 \\
$\quad$ Hypertension & 0.8 & 0.05 \\
$\quad$ Hypercholesterolemia & 1.1 & 0.09 \\
\hline
\end{tabular}

laboratory tests including brain natriuretic peptide (BNP) testing did not reveal any features of cardiac dysfunction, with no electrocardiographic changes in any of the 10 patients.

Paclitaxel cardiotoxicity increased among patients with diabetes mellitus, hypertension, prior chest wall irradiation, ECOG PS 2, age $>60$ years, and metastatic or recurrent disease (table 5).

\section{Discussion}

The cardiotoxicity associated with paclitaxel used to not be well understood, and studies showed contradictory results in comparison with other agents like doxorubicin and trastuzumab. Some studies showed that paclitaxel led to CHF in 5-15\% at conventional doses. Others showed severe reductions in EF in 1-2\% of patients $[13,14,17]$. Del Mastro et al. [18] showed the incidence of cardiotoxicity after weekly paclitaxel to be $9 \%$, with $2.5 \%$ of patients having grade 4 cardiotoxicity.

Paclitaxel cardiotoxicity seems to be mild in most cases. Further, a study in patients with major cardiac risk factors including unstable angina, CHF, and atrial fibrillation showed that paclitaxel can be safely administered as a single therapy or in combination with a noncardiotoxic platinum agent such as cisplatin or carboplatin $[19,20]$.

The current trial's main objective was to evaluate the potential risk of paclitaxel for EF after a relatively long follow-up period. Studies examining cardiac toxicity of other chemotherapies with proven cardiotoxicities like trastuzumab applied the same followup period to assess late cardiac events [21].

The current study agrees with previous reports in that paclitaxel is one of the chemotherapeutic agents to induce cardiotoxicity. The incidence of cardiac toxicity in the current study was $20 \%$, with grades 1 and 2 occurring in 16 and 4\%, respectively. Those figures are slightly higher than those reported by Curigliano et al. [13]. On the other hand, our results were relatively better than those of Del Mastro et al. [18]. The differences between studies may be related to the relatively small number of patients (all trials including ours) and the respective duration of follow-up (2 years in the former and only 6 months in the latter trial). Another explanation may be attributed to the chemotherapy used. While paclitaxel was given in combination with other agents in one trial, it was given as a weekly single agent in the other $[13,18]$.

An important point that may explain the difference between our results and those of previous reports is that cardiotoxic effects were recorded based on regular echocardiographic evaluations in our trial. Regular and routine monitoring was not carried out in other clinical trials; hence similar toxicities may have occurred but were not recorded.

The authors used echocardiography as a method to evaluate changes in EF related to paclitaxel as it is widely available, easily accessible, cheap, and simpler than other methods. Furthermore, it has an overall sensitivity of $50-60 \%$ to detect changes in EF and is applied in all guidelines including those of the British Colombia Cancer Agency (BCCA) [22, 23].

The long-term effects of paclitaxel on EF were examined extensively in the current work. Accordingly, paclitaxel-related cardiotoxicity can occur with conventional doses and is generally mild. It can occur at a relatively lower rate within the first 12 months after treatment rather than more than 12 months of treatment and is not cumulative.

Furthermore, the authors evaluated biochemical markers including BNP and electrocardiographic changes for $\mathrm{CHF}$ in the 10 patients who developed a decline in EF in the follow-up period. Here, we observed no changes associated with the echocardiographic finding of a decline in EF.

Paclitaxel-related cardiotoxicity increased among patients with high-risk features including diabetes mellitus, hypertension, radiotherapy to the chest wall, ECOG PS 2, age > 60 years, and paclitaxel treatment in the metastatic or recurrent setting [24].

The current study has some limitations in that the authors included EF data only (without other echocardiographic data). Additionally, the authors did not perform speckle tracking strain imaging, which we believe might improve the early diagnosis of chemotherapy-induced cardiotoxicity (awaiting further confirmation from clinical trials).

Researchers/clinicians should keep in mind that paclitaxel has some cardiotoxic effects. This may lead to important questions about its application in combination with other cardiotoxic agents like trastuzumab and its use in recurrent platinum-resistant ovarian cancer. More studies are required to confirm our results and find answers to the above questions.

\section{Conclusion}

Paclitaxel has cardiotoxic effects. Careful monitoring of cardiac function during and after paclitaxel infusion is required in patients with high-risk features.

\section{Disclosure Statement}

The authors certify that they have no affiliations with or involvement in any organization or entity with any financial or non-financial interest that might affect the results of the current study. 


\section{References}

1 National cancer institute: dtp.nci.nih.gov/timeline/ flash/success_stories/S2_taxol.htm.

2 Gianni L, Munzone E, Capri G, Villani F, Spreafico C, Tarenzi E, Fulfaro F, Caraceni A, Martini C, Laffranchi A: Paclitaxel in metastatic breast cancer: a trial of two doses by a 3-hour infusion in patients with disease recurrence after prior therapy with anthracyclines. J Nat Cancer Inst 1995;87:1169-1175.

3 Danesi R, Innocenti F, Fogli S, Gennari A, Baldini E Di Paolo A, Salvadori B, Bocci G, Conte PF, Del Tacca M: Pharmacokinetics and pharmacodynamics of combination chemotherapy with paclitaxel and epirubicin in breast cancer patients. Br J Clin Pharmacol 2002;53. 508-518.

4 Clin-Info: e-Compendium of Pharmaceuticals and Specialties. www.pharmacists.ca/products-services/ compendium-of-pharmaceuticals-and-specialties/.

5 Niederhuber JE: Global Cancer Control: An Essential Duty. NCI Cancer Bulletin, NCI, 2009

6 Khakoo AY, Liu PP, Force T, Lopez-Berestein G, Jones LW, Schneider J, Hill J: Cardiotoxicity due to cancer therapy. Tex Heart Inst J 2011;38:253-256.

7 Bergler-Klein J: Strain and left ventricular volumes for predicting cardiotoxicity: a life-saving approach in anthracycline cancer treatment. Eur Heart J Cardiovasc Imaging 2015;16:968-969.

8 Dolci A, Dominici R, Cardinale D, Sandri MT, Panteghini M: Biochemical markers for prediction of chemotherapy-induced cardiotoxicity: systematic review of the literature and recommendations for use Am J Clin Pathol 2008;130:688-695.

9 Herrmann J, Yang EH, Iliescu CA, Cilingiroglu M, Charitakis K, Hakeem A: Vascular toxicities of cancer therapies: the old and the new - an evolving avenue. Circulation 2016;133:1272-1289.
10 Schimmel KJ, Richel DJ, van den Brink RB, Guchelaar HJ: Cardiotoxicity of cytotoxic drugs. Cancer Treat Rev 2004:30:181-191.

11 Ederhy S, Cohen A, Dufaitre G, Izzedine H, Massard C, Meuleman C, Besse B, Berthelot E, Boccara F, Soria JC: QT interval prolongation among patients treated with angiogenesis inhibitors. Target Oncol 2009;4:89-97.

12 Lainscak M, Dagres N, Filippatos GS, Anker SD, Kremastinos DT: Atrial fibrillation in chronic non-cardiac disease: where do we stand? Int J Cardiol. 2008;128: 311-315.

13 Curigliano G, Mandala M, Cipolla CM: Cancer treatment in patients with heart disease, chapter 8; in Ballová V, Pulla M (eds): ESMO Handbook of cancer treatment in special clinical situations. ESMO Press, 2013.

14 Curigliano G, Mayer EL, Burstein HJ, Winer EP, Goldhirsch A: Cardiac toxicity from systemic cancer therapy: a comprehensive review. Prog Cardiovas Dis 2010;53:94-104.

15 NCCN clinical practice guidelines in oncology, ovarian cancer, version 1, 2013.www.nccn.org/professionals/ physician_gls/pdf/ovarian.pdf.

16 Common Toxicity Criteria (CTC) Version 2.0. www. eortc.be/services/doc/ctc/ctcv20_4-30-992.pdf; published 30 April 1999.

17 Bristol-Myers Squibb Canada: TAXOL ${ }^{\circledR}$ product monograph. Montreal, Ontario, 22 February 2010.

18 Del Mastro L, Perrone F, Repetto L, Manzione L, Zagonel V, Fratino L: Weekly paclitaxel as first-line chemotherapy in elderly advanced breast cancer patients: a phase II study of the Gruppo Italiano di Oncologia Geriatrica (GIOGer). Ann Oncol 2005;16:253-258.

19 Eisenhauer EA, Vermorken J: The taxoids. Comparative clinical pharmacology and therapeutic potential. Drugs 1998;55:5-30
20 Baldini E, Prochilo T, Salvadori B, Bolognesi A, Aldrighetti D, Venturini M, Rosso R, Carnino F, Gallo L, Giannessi P, Conte PF, Orlandini C, Bruzzi P: Multicancer randomized phase III trial of epirubicin plus paclitaxel vs epirubicin followed by paclitaxel in metastatic breast cancer patients: focus on cardiac safety. $\mathrm{Br}$ J Cancer 2004;91:45-49.

21 Romond EH, Jeong JH, Rastogi P, Swain SM, Geyer CE Jr, Ewer MS, Rathi V, Fehrenbacher L, Brufsky A, Azar CA, Flynn PJ, Zapas JL, Polikoff J, Gross HM, Biggs DD, Atkins JN, Tan-Chiu E, Zheng P, Yothers G, Mamounas EP, Wolmark N: Seven-year follow-up assessment of cardiac function in NSABP B-31, a randomized trial comparing doxorubicin and cyclophosphamide followed by paclitaxel (ACP) with ACP plus trastuzumab as adjuvant therapy for patients with node-positive, human epidermal growth factor receptor 2-positive breast cancer. J Clin Oncol 2012;30:3792-3799.

22 Gaber R, Kotb NA, Ghazy M, Nagy HM, Salama M, Elhendy A: Tissue Doppler and strain rate imaging detect improvement of myocardial function in iron deficien patients with congestive heart failure after iron replacement therapy. Echocardiography 2012;29:13-18.

23 BCCA Protocol summary for adjuvant therapy for breast cancer using trastuzumab (Herceptin) following the completion of chemotherapy (sequential). $w w w \cdot b c$ cancer.bc.ca/chemotherapy-protocols-site/Documents/ Breast/BRAJTR_Protocol_1Aug2016.pdf.

24 Zamorano JL, Lancellotti P, Muñoz DR, Aboyans V, Asteggiano R, Galderisi M, Habib G, Lenihan DJ, Lip GY, Lyon AR, Fernandez TL, Mohty D, Piepoli MF, Tamargo J, Torbicki A, Suter TM: 2016 ESC position paper on cancer treatments and cardiovascular toxicity developed under the auspices of the ESC Committee for Practice Guidelines. Eur Heart J 2016;37:2768-2801. 